\title{
Metformin Resistant MDA-MB-468 Cells Exhibit EMT-like Phenotype and Increased Migration Capacity
}

Sahika Cingir Koker ( $\nabla$ sahika.koker@gmail.com )

Ufuk University: Ufuk Universitesi https://orcid.org/0000-0002-8529-4532

\section{Banu Yalcin}

Ufuk University: Ufuk Universitesi

Irem Dogan Turacli

Ufuk University: Ufuk Universitesi

Research article

Keywords: Metformin, Breast Cancer, EMT, Drug Resistance, PI3K inhibitor

Posted Date: April 8th, 2021

DOl: https://doi.org/10.21203/rs.3.rs-382083/v1

License: (c) (1) This work is licensed under a Creative Commons Attribution 4.0 International License.

Read Full License 


\section{Abstract}

Background: Metformin is one of the most prescribed drug for the treatment of type II diabetes. Its antiproliferative effect is also taken advantage for the treatment of cancer. Despite many of the studies mention the positive effects of metformin in inhibiting the proliferation of cancer cells, there are also studies which questions this idea as well.

Methods: In this study, we investigated the most widely studied breast cancer cell lines, ER(+) MCF7 cells, TNBC MDA-MB-231 and MDA-MB468 cells in terms of metastatic behavior under long-term metformin treatment. MCF7, MDA-MB231 and MDA-MB468 cells were gained resistant to metformin starting from $0.2 \mathrm{mM}$ to $3.2 \mathrm{mM}$.

Results: Compared to MCF7 and MDA-MB231 cell lines, we only observed dramatic changes in MDAMB468 cells whose morphology has been changed towards mesenchymal like phenotype. Moreoever, migration capacity of these cells were also significanlty increased which were validated at both mRNA and protein levels as well as wound healing assay. In addition EMT like phenotype and increasing migration capacity of metformin resistant MDA-MB468 cells, they exhibited less sensitivity to PI3K inhibitor.

Conclusions: All together, our data pointed out that, metformin's effects should be questioned depending on the subtype of the breast cancer that's to be treated.

\section{Background}

Insulin resistance and hyperinsulinemia are well known risk factors for cancer development [1]. Besides, oxidative stress due to hyperinsulinemia, accumulation of glycation end products is the enhancers of tumor progression. Metformin is a widely prescribed drug for the treatment of type 2 diabetes (T2D) which helps to decrease hepatic gluconeogenesis and increase peripheral insulin sensitivity by enhancing glucose uptake in muscle and adipose tissues. Interestingly, several preclinical and clinical studies reveal that metformin use is associated with the inhibition of cancer incidence and mortality $[2,3]$.

According to the mechanistic studies which are evaluating the current evidence for associations between metformin use and anti-tumorigenic effects, cancer risk and survival outcomes usually pointed out the mitochondrial electron transport chain. Complex I inhibition by metformin interrupts mitochondrial oxidative phosphorylation that is the main cellular energy mechanism of a living cell. It causes a decrease in ATP synthesis and leads to increased AMP/ATP ratio. Elevated AMP binds to the energy sensor of the cell, 5'-AMP-activated protein kinase (AMPK), that is affected by this change and gets activated. The activated AMPK plays important roles in regulating lipid and protein metabolisms by phosphorylating a series of target proteins. AMPK can inhibit gluconeogenesis and activates glycolysis in liver. Also, its activation leads to glucose consumption in the muscle tissue [4-6]. Besides, persistent activation of AMPK can cause cytostatic and even cytotoxic effects [7]. As another mechanism, the increase in insulin levels has been shown to enhance free or active insulin-like growth factor (IGF-1) and 
decrease IGF- binding proteins (IGFBP), resulting in the activation of IGF-1R signaling which in turn activates Phosphatidylinositol-3-kinase (PI3K) and mitogen-activated protein kinase (MAPK) signaling. Metformin can reduce the levels of insulin and IGF-1, which can lead to inhibition of cancer cell growth [8].

Metformin, as a glucose and insulin lowering agent, can cut off the main energy supply of a cancer cell. Although it is known that cancer cells preferentially metabolize glucose to lactate, studies of tumor metabolism in vivo have suggested that some tumor cells also prefer oxidative phosphorylation in the mitochondria [9]. Since metformin disturbs mitochondrial complex I, it is effective on cancer cell's energy metabolism which may lead to cell death. However, the concentration used in vitro, is very high to be translated to in vivo. Thus, it is controversial to see the same anticarcinogenic effects in tumor tissues. Likewise, in a study done by Sadighi et al., they observed that metformin did not exhibit cytotoxicity in various breast cancer cell lines, when it is used at pharmacological concentration and normal plasma glucose levels [10].In addition to this, when metformin is used under high glucose conditions which mimics the hyperglycemic condition, the effect of metformin was reduced in triple negative breast cancer cell lines (TNBC) [11].

Most of the epidemiological studies demonstrated a connection between diabetes and breast cancer (BC). Of particular interest is metformin generally associated with a decreased risk of cancer in diabetes patients. Specifically, a decreased risk of breast cancer was observed in women with T2D using metformin on a long-term basis compared with other antidiabetic drugs [12]. However, Currie et al demonstrated that metformin use was associated with lower risk of colon and pancreas cancer but did not affect the risk of breast or prostate cancer [13]. In a cohort study, metformin users are associated with lengthened survival, decreased breast cancer-specific mortality than non-users in diabetics with HER2 + breast cancer [14]. Bayraktar et al. showed that TNBC patients using metformin tend to have a lower risk of distant metastases compared to those not taking metformin [15]. Also, Jacob et al showed that the use of metformin in women with diabetes and breast cancer may reduce the risk of metastases [16]. However, in a very recent study conducted by Park et, they revealed that while the long-term metformin usage may reduce the risk of ER (+) BC, it increased the risk of developing ER (-) breast cancer and TNBC [17].

Despite the success of treatments, many cancers eventually adapt to medications, and this resistance is a primary cause of tumor progression and death. Tumors are either intrinsically drug resistant or acquire resistance at some point during multiple courses of therapy [18]. Genetic and epigenetic factors of the tumor can determine the drug sensitivities within the subpopulations of tumor cells [19]. Tumor subpopulations selected for drug resistance can be more aggressive than their drug-sensitive counterparts. This drug resistant behavior can favor metastatic program which is composed of loss of cellular adhesion, invasion of surrounding tissue, intravasation and survival in the circulation, extravasation through vascular walls into the parenchyma of distant tissues and formation of metastatic colonies [20]. These evolutionary advantageous metastatic populations can emerge under the selective pressure of environmental circumstances such as therapy resistance [21]. 
In this study, we aim to evaluate the changes on metastatic profile of breast cancer cell lines which have different genomic background (Table 1) under long term-increasing metformin pressure. The complex interplay between the breast cancer cells, their environment and drug resistance can influence tumor progression and metastasis which can provide insight to understand metastasis in breast cancer.

\section{Methods}

\section{Generation of Metformin Resistant Cells}

In this study representative of breast cancer cell lines, MCF7 (kind gift by Dr. Işık Yuluğ), MDA-MB-468 (kind gift by Dr. Özgür Şahin) and MDA-MB-231 (kind gift by Dr. Özlen Konu) were used. As a starting point, $0.2 \mathrm{mM}$ Metformin (Merck cat:317240) was added to complete DMEM. Each following week, the concentration of metformin was doubled until $3.2 \mathrm{mM}$ concentration has been reached.

\section{Cell Viability Assay}

$5 \times 10^{3}$ MDA-MB-468 cells were seeded in 96-well plates and next day cells were treated with or without 10mM Metformin or $10 \mu \mathrm{M}$ PI3K inhibitor (LY294002-Cell Signaling Technology-CST- cat.no:9901P) or $10 \mathrm{mM}$ Metformin together with $10 \mu \mathrm{M}$ PI3K inhibitor. At the end of each time point -either 24hour or 48hour- MTT (3- (4,5-Dimethyltiazol-2-yl)-2,5-Diphenyltetrazolium Bromide), (Merck cat: 475989) assay was performed based on manufacturer's instructions. Color absorbance was measured using microplate reader (SpectraMax iD3) at a wavelength of $570 \mathrm{~nm}$. Results were normalized against the mean measurements from at least six replicates which were not exposed to any treatment. Each experiment was repeated twice.

\section{Rna Isolation, Cdna Synthesis And Rt-qpcr}

RNA was isolated from the cell pellets by using GENEALL RNA isolation kit (Catalog number: 305 - 101) according to the manufacturer's protocol and RNA's were transcribed into cDNA by using Roche Transcriptor High Fidelity cDNA (Complementary DNA) Synthesis Kit (Catalog number: 5091284001). Followed by cDNA synthesis, RT-qPCR (Real Time Polymerase Chain Reaction) was done by using SYBR green (Biorad) with Biorad CFX Connect Real Time System. Beta Actin was used as a reference gene. Graphs were plotted by using log2 fold changes for each gene which were normalized against control group. Primers used in this study are listed in the additional file 1.

\section{Western Blot}

Western Blot 
At the end of a week for each indicated concentration, cell pellets were obtained from the cells which were seeded $2 \times 10^{5}$ per 6 well plate. Proteins were isolated by using these pellets with freshly prepared RIPA buffer ( $\mathrm{NaCl}$, TrisHCl, NP-40, 10\% SDS, protease inhibitor Coctail (CST 5871S) and phosphatase inhibitor cocktail (CST 5870S). With BCA protein assay reagent Kit (Thermo Scientific, USA,23227), total protein concentrations were determined. Before loading to the gel, proteins were denatured with 4X Loading Dye (Biorad, USA, 161-0747) at $95{ }^{\circ} \mathrm{C}$ for 5 minutes. Proteins were run in 10\% SDS-PAGE (161-0183, Bio-Rad Acrylamide Kit) and then transferred onto PVDF membrane (Merck ref.no: 3010040001) by semi-dry transfer method. At the end of transfer, membranes were blocked with $5 \%$ milk prepared with Trisbuffered saline with Tween-20 (0,2\%) (TBST) for $1 \mathrm{~h}$ at room temperature. Then, membranes were incubated with the following antibodies at $4^{\circ} \mathrm{C}$ overnight at a dilution 1:1000 if not indicated otherwise. At the end of 24 hour, membranes were first washed 3 times for 10 minutes with TBST, and then they were incubated with secondary antibodies (1:5000) for $1 \mathrm{~h}$ at room temperature. Then, they were again washed 3 times for 10 minutes with TBST. Indicated proteins were detected by using ECL (Biorad cat.no:170062) and images were captured with Biorad ChemiDoc XRS+. Beta-Actin was used as a loading control. The antibodies used in this study are Beta Actin (CST 4967), Slug (CST 9585), Beta Catenin (CST 9587), ECadherin (CST 3195), N-Cadherin (CST 13116), ZEB1 (CST 3396T), Claudin (CST 13255), Snail (CST 3879), MMP2 (CST 878095), Vimentin (CST 5741).

\section{Wound Healing Assay:}

To assess the migration capacity of MDA-MB-468 cells which were resistant to different concentrations of metformin were seeded ( $2 \times 10^{5}$ cells). Next day with 200 ul sterile pipette tip, vertical wounds were introduced then their images were taken with Olympus CKX53. At the end of 24 hour, second images were taken as well. Each time at least 6 images were taken. The distance between the two sides of the wound were measured blindly with the use of Olympus cellSens Entry software.

\section{Statistics}

Graphpad (Prism 8) was used to plot the graphs and for statistical analysis. For RT-qPCR, log2 of $\Delta \Delta C t$ values were plotted and analyzed with One-Way ANOVA with multiple comparisons (Dunnett's multiple comparison tests). MTT results were plotted by using relative percentages of cell viability to the control group and analyzed by using Two-Way-ANOVA with multiple comparison tests (Tukey' multiple comparison tests). For wound healing assay, the graphs were generated by using the relative percentages of migration to the control group and analyzed by using Two-Way-ANOVA with multiple comparison tests (Sidak's multiple comparison tests).

\section{Results}

Metformin results in changes in gene expression levels in breast cancer cells 
MDA-MB 468, MDA-MB 231 and MCF7 cells were treated with increasing doses of metformin $(0,2 ; 0,4 ; 0,8$; 1,$6 ; 3,2 \mathrm{mM}$ ) in long term that they were incubated in each dose longer than a week. Then, we first analyzed the changes in transcriptional profile in that long-term metformin treated cells to see whether any cellular process was affected by the drug. The qPCR data showed that PIK3CA and mTOR mRNA expressions were downregulated in all cell lines. Even though $0,4 \mathrm{mM}$ and $0,8 \mathrm{mM}$ long term metformin treated MDA-MB-468 cells did not change the PIK3CA expressions significantly, PIK3CA was significantly downregulated in all other cells and in all treatment groups. Accordingly, we observed statistically significant downregulation pattern in mTOR gene expressions in long term metformin treated cells excluding 0,4 mM metformin treated MDA-MB 468 cells and 0,8 mM and 1,6 mM treated MDA MB 231 cells. Interestingly, TOP2A and CCND1 gene expression levels exhibited increasing pattern in MDA-MB468 cells which was the opposite of what was observed in MDA-MB231 and MCF7 cells. Whereas ZEB1, Vimentin and MMP9 expressions were upregulated in long term metformin treated MDA-MB 468 cells, that was not the case in the other cell lines. In MDA-MB 468 cells long term metformin treatment induced significant ZEB1 and VIM expression in all concentrations. Also, MMP9 was upregulated significantly in $0,4 \mathrm{mM} ; 1,6 \mathrm{mM}$ and 3,2 mM metformin groups in MDA-MB-468 cells (Fig. 1).

\section{The morphological changes are related with long term metformin treatment in a dose dependent manner}

As metformin appears to induce proliferation and epithelial-mesenchymal transition (EMT) related gene expressions, we examined morphological changes in long term metformin treated MDA-MB 468 cells (will be referred to as metformin resistant [met-R] cells thereafter). As the cells got resistance to increasing doses of metformin, they lost their sphere like shape, and instead they gained spindle-like appearance (Fig. 2A). Especially the $1,6 \mathrm{mM}$ and the 3,2 mM metformin resistant cells exhibited mesenchymal phenotype which leaded us to investigate further the migratory capacity of these cells.

\section{Metformin resistant cells exhibit significantly higher migratory capacity in a dose dependent manner}

In the wound healing assay, we examined cell migration capacity of metformin resistant MDA-MB 468 cells by introducing mechanical scratch. Images of scratch areas from the time points 0 and 24 hours are illustrated in Fig. 2b. Wound healing assay revealed that high dose metformin resistant MDA-MB 468 cells had significantly more migration capacity compared to low dose resistant cells (Fig. 2B).

\section{Metformin resistant MDA-MB 468 cells are involved in metastatic processes}

The observation of increased migration capacity of these met-R MDA-MB -468 cells led us to investigate whether long term metformin use can also lead to EMT in TNBC. We tested this possibility by examining EMT related protein expressions in metformin resistant concentrations in MDA-MB 468 cells. As shown in Fig. 3, Zeb1, N-cadherin, Vimentin and Slug protein expressions were upregulated in all resistant concentrations. Accordingly, Claudin expression was downregulated in metformin resistant groups compared to parental control which demonstrates metastatic changes are compatible with microscopic images. Also, E-cadherin expression was downregulated in all resistant concentration groups except 0,4 $\mathrm{mM}$ metformin. While b-catenin was decreasing, snail was enhanced in $0,2 \mathrm{mM}, 1,6 \mathrm{mM}$ and 3, $2 \mathrm{mM}$ 
metformin resistance cells. These distinct changes in EMT markers, clearly demonstrates that metformin resistant MDA-MB 468 cells has metastatic capacity compared to their parental counterparts.

\section{Effects of metformin and LY294002 on cell viability in metformin resistant MDA-MB 468 cells}

To check the proliferation pattern of metformin resistant MDA-MB-468 cells, MTT assay was used. Moreover, to assess whether metformin resistance will affect those cells' sensitivity to PI3K inhibitor, we included PI3K inhibitor (LY294002) to the experimental set up as well (Fig. 4). We aimed to observe whether IC50 concentration of metformin induced cell death in metformin resistant MDA-MB 468 cells or not. As shown in Fig. 4, 10 mM metformin treatment was not effective on cell death in both 24 and 48 hours in all resistant groups compared to the control (parental) group. To determine the antiproliferative effects of a selective phosphatidylinositol 3-kinase (PI3K) inhibitor LY294002, all cells were treated with IC50 dose (10u) of LY294002 for 24 and 48 hours with or without metformin. After 24-hour treatment, 10 uM LY294002 exhibited higher cell proliferation significantly in 0,2mM, 0,4mM, 0,8mM metformin resistant MDA-MB468 cells compared to their control group. Also, at 48 hours, 10 uM LY294002 was significantly less effective in 0.2 and $3.2 \mathrm{mM}$ met-R cells in terms of reducing cell proliferation. Combined treatments led to a further decrease in cell viability compared to alone treatments of these agents. Therefore, these two agents have synergistic effects on reduction of metformin resistant MDA-MB 468 cell proliferation. However, the cell viability was lower in combinatorial treatment groups in metformin resistant cells compared to their parental controls. Especially, 0,2 $\mathrm{mM}$ at 24 hour and 3,2 $\mathrm{mM}$ metformin resistant MDA-MB 468 cells at 48 hours had significantly higher proliferation rates in combined treatments compared to control groups (Fig. 4).

\section{Discussion}

Metformin, a well-tolerated diabetic insulin sensitizer, has been associated with decreased risk of cancer in diabetic patients. Of particular interest, the type 2 diabetic women taking metformin have reduced the risk of breast cancer [12] and also tend to have lower risk of metastases in TNBC patients compared to those not taking this drug [15]. However, the reduced metastasis risk in metformin treated TNBC patients in Bayraktar et al study was not significant which needed to be point out carefully. In that cohort study, which was comprised of 63 diabetic patients receiving metformin, there were 67 diabetic patients not receiving metformin, and 1318 nondiabetic patients with no information of disease period and also duration of metformin use [15]. The tendency to the inhibition of metastasis in metformin receiving patients may due to mentioned limitations above and also short term usage of the drug. Also, Jabob et al demonstrated that metformin significantly decreased the risk of metastases in breast cancer patients without specifying TNM classification, hormone receptor status and preventive target therapy treatment [16].

Liver kinase 1 (LKB1 also known as STK11) is a tumor suppressor gene that is inactivated by bi-allelic mutation in several cancer types. Metformin inhibits mitochondria complex I, that leads to the depletion of intracellular ATP and enhancing AMP levels. AMP can bind to the regulatory domain of AMPK and 
promotes its phosphorylation by LKB1 [22]. This LKB1/AMPK interaction is important in protecting AMPK, which can be dephosphorylated by cellular phosphatases [23]. Therefore, metformin causes AMPK dependent growth inhibition that requires LKB1. Nevertheless, it has been shown that metformin leads to cell death in LKB1 null cells which can be commentated as metformin caused cell death in an AMPK independent manner [24]. It has been shown that Phenformin, a biguanide and an analog of metformin, induced cell death in LKB1 mutant non-small cell lung cancer cells [24]. The lack of proper energy consumption results in cell death in LKB1 absence, thus it can be suggested as LKB1 activation can promote cell survival against phenformin [25]. In our study we included three of the most representative breast cancer cell lines (MDA-MB-468, MDA-MB-231 and MCF7) and observed metastatic effects of long term metformin usage in MDA-MB 468 but not in MCF-7 and MDA-MB 231 cells. When we analyze the genotypic differences between MDA-MB 468 and MDA-MB 231 TNBC cells, it was observed that the LKB1 status was different. The absence of LKB1 in MDA-MB 231 cells may cause cell death which are hypersensitive to metabolic stress caused by biguanides.

The PI3K/AKT signaling pathway is one of the best targets for breast cancer treatment [26]. When PI3K is inhibited, the corresponding expressions of downstream effector proteins are reduced, and cell proliferation is inhibited [27]. However, in our study, PI3K inhibitor LY29004 did not inhibit proliferation in Metformin resistant cells. When concrescence with metastatic profiles of met-resistant MDA-MB468 cells, it can be interpreted as the aggressive behavior of these cells overcome the effects of PI3K inhibition and did not sensitize cells to LY29004.

It is a very well known fact that there are genomic differences between cancer cell lines [28]. In the literature there are many studies showing the relationship between the molecular profiles of breast cancer cell lines and their relation with invasiveness. Thompson et al. showed that MCF7 cells defined as ER (+), Vimentin (-), while MDA-MB-231 cells defined as ER(-) and Vimentin (+) whereas MDA-MB-468 cells were presented as ER (-), VIM (-) [29]. In our study, we also did not observe vimentin expression in parental MDA-MB-468 cells. However long term metformin exposure clearly reprogrammed the genetic profile of these cells, starting even from the lowest concentration until to the highest doses of metformin. We also observed increased expression of vimentin in both mRNA and protein levels in MDA-MB468 cells. Loss of ER as in the case of MDA-MB468 cells, and gain of Vimentin expression, in which we achieved with long term metformin exposure, also associated with increased metastatic potential [29]. Therefore the difference that we have observed between MCF7 cells and MDA-MB-468 cells may come from ER status of these cell lines. As stated by Thompson et al, the absence or loss of ER by itself may not linked to increased invasiveness however, expression of Vimentin together with the absence of ER associated with increased invasiveness as we have also observed with ER (-) MDA-MB-468 cells but not with ER (+) MCF7 cells [29]. In the same study where they also studied the Vimentin expression in MCF7 cells and MCF7ADR (adriamycin resistant) cell, gaining resistance to AR completely reversed the expression of Vimentin where they gain its expression. By relying on this phenomena, and known the fact that Vimentin expression is an acquireable trait [29], we hypothise that as MDA-MB-231 cells gain resistance to metformin, they completely reversed their Vimentin profile by losing its expression compared to parental MDA-MB-231 cells. Moreover, in a recent study where Banerjee et al. kept MDA-MB-231 cells with 2mM 
Metformin longer than 8 weeks, they observed that elongated fibroblastoid appearance of MDA-MB-231 had not been changed [30].

In the course of treatment of any disease, resistance can develop for any drug and metformin is not an exception [31]. Short term treatment of metformin in breast cancer patients can provide benefits [32] however, long term usage together with proper classification of breast cancer tumor type to be assigned for metformin usage should be addressed carefully. Park et al. examined the link between metformin use, T2D and breast cancer patients classified according to hormone receptor status. They observed decreased risk of developing ER (+) breast cancer associated with T2D using metformin, however they observed increased risk of development of ER (-) breast cancer and TNBC associated with T2D using metformin [17].

In a study done by Ferraros et al, they generated metformin resistant MCF7 cells and with total transcriptome analysis and revealed that metformin treatment was resulted in reprogramming of their transcriptome towards a metastatic stem-like profile [33]. This observation was compatible with what we observed with LKB1 wt, MDA-MB-468 cells which is a TNBC cell line. However, we could not reach the same observation with MCF7 cells which might resulted from difference in resistance concentration between that study and in our study.

\section{Conclusions}

In this study, LKB1 wt, TNBC MDA-MB-468 cells exhibited EMT-like phenotype compared to ER (+) MCF7 cells and LKB1 mutant MDA-MB-231 TNBC cells upon long term metformin exposure. It is certain that more studies are required to reveal the reasons behind the differences occurring among breast cancer subtypes. Although our results show a clear connection between metformin and metastasis in TNBC, it will be more informative to see the micro-environmental and metastatic changes in long term metformin treatment in vivo TNBC model in the future. Moreover, when the genes exhibited changes in expression profile along with long term metformin treatment were investigated in miRNET online bioinformatics tool [34], there are shared microRNAs affected by these genes (Fig. 5). Therefore, observed changes could also be attributed to the changes in the expression profile of these miRNAs under long term metformin treatment. As a future perspective, analysis of selective miRNAs between the met-R cells who are resistant to different concentrations of metformin could also provide valuable information to understand the mechanism behind EMT like phenotype that was observed with met-R MDA-MB-468 cells.

\section{Abbreviations}

T2D

Type 2 diabetes

AMPK

5'-AMP-activated protein kinase

IGF-1 
Insulin-like growth factor

IGFBP

IGF- binding proteins

$\mathrm{PI} 3 \mathrm{~K}$

Phosphatidylinositol-3-kinase

MAPK

Mitogen-activated protein kinase

TNBC

Triple negative breast cancer cell lines

BC

Breast cancer

CDNA

Complementary DNA

RT-qPCR

Real Time Polymerase Chain Reaction

TBST

Tris-buffered saline with Tween-20 (0,2\%)

EMT

Epithelial-mesenchymal transition

met-R

Metformin resistant

LKB1

Liver kinase 1

MCF7-ADR

Adriamycin resistant MCF7 cells

\section{Declarations}

Ethics approval and consent to participate: Not Applicable

Consent for publication: Not Applicable

Availability of data and materials: All data generated or analysed during this study are included in this published article [and its supplementary information files].

Competing interests: The authors declare that they have no competing interests.

Funding: Ufuk University

Authors' contributions: IDT designed the study. SCK, BY and IDT conducted the experiments. SCK and IDT wrote the manuscript. All authors read and approved the study. 
Acknowledgements: The authors are grateful to Mrs. Naime Tömek for the establishment of ONTAL where the studies are conducted.

\section{References}

1. Smith U, Gale EA. Cancer and diabetes: are we ready for prime time? Diabetologia 2010, 53(8):15411544.

2. Evans JM, Donnelly LA, Emslie-Smith AM, Alessi DR, Morris AD. Metformin and reduced risk of cancer in diabetic patients. BMJ. 2005;330(7503):1304-5.

3. Gandini S, Puntoni M, Heckman-Stoddard BM, Dunn BK, Ford L, DeCensi A, Szabo E. Metformin and cancer risk and mortality: a systematic review and meta-analysis taking into account biases and confounders. Cancer Prev Res (Phila). 2014;7(9):867-85.

4. Luengo A, Sullivan LB, Heiden MG. Understanding the complex--1ty of metformin action: limiting mitochondrial respiration to improve cancer therapy. BMC Biol. 2014;12:82.

5. Mihaylova MM, Shaw RJ. The AMPK signalling pathway coordinates cell growth, autophagy and metabolism. Nat Cell Biol. 2011;13(9):1016-23.

6. Hardie DG. The LKB1-AMPK pathway-friend or foe in cancer? Cancer Cell. 2013;23(2):131-2.

7. Li W, Saud SM, Young MR, Chen G, Hua B. Targeting AMPK for cancer prevention and treatment. Oncotarget. 2015;6(10):7365-78.

8. Kourelis TV, Siegel RD. Metformin and cancer: new applications for an old drug. Med Oncol. 2012;29(2):1314-27.

9. Marin-Valencia I, Yang C, Mashimo T, Cho S, Baek H, Yang XL, Rajagopalan KN, Maddie M, Vemireddy V, Zhao Z, et al. Analysis of tumor metabolism reveals mitochondrial glucose oxidation in genetically diverse human glioblastomas in the mouse brain in vivo. Cell Metab. 2012;15(6):827-37.

10. Sadighi S, Amanpour S, Behrouzi B, Khorgami Z, Muhammadnejad S. Lack of metformin effects on different molecular subtypes of breast cancer under normoglycemic conditions: an in vitro study. Asian Pac J Cancer Prev. 2014;15(5):2287-90.

11. Varghese S, Samuel SM, Varghese E, Kubatka P, Busselberg D. High Glucose Represses the AntiProliferative and Pro-Apoptotic Effect of Metformin in Triple Negative Breast Cancer Cells. Biomolecules 2019, 9(1).

12. Bodmer M, Meier C, Krahenbuhl S, Jick SS, Meier CR. Long-term metformin use is associated with decreased risk of breast cancer. Diabetes Care. 2010;33(6):1304-8.

13. Currie CJ, Poole CD, Gale EA. The influence of glucose-lowering therapies on cancer risk in type 2 diabetes. Diabetologia. 2009;52(9):1766-77.

14. He X, Esteva FJ, Ensor J, Hortobagyi GN, Lee MH, Yeung SC. Metformin and thiazolidinediones are associated with improved breast cancer-specific survival of diabetic women with HER2 + breast cancer. Ann Oncol. 2012;23(7):1771-80. 
15. Bayraktar S, Hernadez-Aya LF, Lei X, Meric-Bernstam F, Litton JK, Hsu L, Hortobagyi GN, GonzalezAngulo AM. Effect of metformin on survival outcomes in diabetic patients with triple receptornegative breast cancer. Cancer. 2012;118(5):1202-11.

16. Jacob L, Kostev K, Rathmann W, Kalder M. Impact of metformin on metastases in patients with breast cancer and type 2 diabetes. J Diabetes Complications. 2016;30(6):1056-9.

17. Park YM, Bookwalter DB, O'Brien KM, Jackson CL, Weinberg CR, Sandler DP. A prospective study of type 2 diabetes, metformin use, and risk of breast cancer. Ann Oncol. 2021;32(3):351-9.

18. Fouad YA, Aanei C. Revisiting the hallmarks of cancer. Am J Cancer Res. 2017;7(5):1016-36.

19. Sabnis AJ, Bivona TG. Principles of Resistance to Targeted Cancer Therapy: Lessons from Basic and Translational Cancer Biology. Trends Mol Med. 2019;25(3):185-97.

20. Lambert AW, Pattabiraman DR, Weinberg RA. Emerging Biological Principles of Metastasis. Cell. 2017;168(4):670-91.

21. Fares J, Fares MY, Khachfe HH, Salhab HA, Fares Y. Molecular principles of metastasis: a hallmark of cancer revisited. Signal Transduct Target Ther. 2020;5(1):28.

22. Oakhill JS, Chen ZP, Scott JW, Steel R, Castelli LA, Ling N, Macaulay SL, Kemp BE. beta-Subunit myristoylation is the gatekeeper for initiating metabolic stress sensing by AMP-activated protein kinase (AMPK). Proc Natl Acad Sci U S A. 2010;107(45):19237-41.

23. Sanders MJ, Grondin PO, Hegarty BD, Snowden MA, Carling D. Investigating the mechanism for AMP activation of the AMP-activated protein kinase cascade. Biochem J. 2007;403(1):139-48.

24. Shackelford DB, Abt E, Gerken L, Vasquez DS, Seki A, Leblanc M, Wei L, Fishbein MC, Czernin J, Mischel PS, et al. LKB1 inactivation dictates therapeutic response of non-small cell lung cancer to the metabolism drug phenformin. Cancer Cell. 2013;23(2):143-58.

25. Zhou W, Zhang J, Marcus Al. LKB1 Tumor Suppressor: Therapeutic Opportunities Knock when LKB1 Is Inactivated. Genes Dis. 2014;1(1):64-74.

26. Ellis H, Ma CX. PI3K Inhibitors in Breast Cancer Therapy. Curr Oncol Rep. 2019;21(12):110.

27. Torbett NE, Luna-Moran A, Knight ZA, Houk A, Moasser M, Weiss W, Shokat KM, Stokoe D. A chemical screen in diverse breast cancer cell lines reveals genetic enhancers and suppressors of sensitivity to PI3K isoform-selective inhibition. Biochem J. 2008;415(1):97-110.

28. Liu K, Newbury PA, Glicksberg BS, Zeng WZD, Paithankar S, Andrechek ER, Chen B. Evaluating cell lines as models for metastatic breast cancer through integrative analysis of genomic data. Nat Commun. 2019;10(1):2138.

29. Thompson EW, Paik S, Brunner N, Sommers CL, Zugmaier G, Clarke R, Shima TB, Torri J, Donahue S, Lippman ME, et al. Association of increased basement membrane invasiveness with absence of estrogen receptor and expression of vimentin in human breast cancer cell lines. J Cell Physiol. 1992;150(3):534-44.

30. Banerjee A, Birts CN, Darley M, Parker R, Mirnezami AH, West J, Cutress RI, Beers SA, Rose-Zerilli MJJ, Blaydes JP. Stem cell-like breast cancer cells with acquired resistance to metformin are sensitive to 
inhibitors of NADH-dependent CtBP dimerization. Carcinogenesis. 2019;40(7):871-82.

31. Hatoum D, McGowan EM. Recent advances in the use of metformin: can treating diabetes prevent breast cancer? Biomed Res Int. 2015;2015:548436.

32. Niraula S, Dowling RJ, Ennis M, Chang MC, Done SJ, Hood N, Escallon J, Leong WL, McCready DR, Reedijk $\mathrm{M}$, et al. Metformin in early breast cancer: a prospective window of opportunity neoadjuvant study. Breast Cancer Res Treat. 2012;135(3):821-30.

33. Oliveras-Ferraros C, Vazquez-Martin A, Cuyas E, Corominas-Faja B, Rodriguez-Gallego E, FernandezArroyo S, Martin-Castillo B, Joven J, Menendez JA. Acquired resistance to metformin in breast cancer cells triggers transcriptome reprogramming toward a degradome-related metastatic stem-like profile. Cell Cycle. 2014;13(7):1132-44.

34. Chang L, Zhou G, Soufan O, Xia J. miRNet 2.0: network-based visual analytics for miRNA functional analysis and systems biology. Nucleic Acids Res. 2020;48(W1):W244-51.

\section{Tables}

Due to technical limitations, table 1 is only available as a download in the Supplemental Files section.

\section{Figures}


A) PI3K Expression
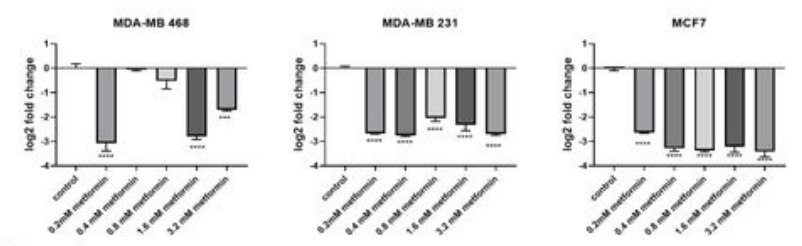

B) $\mathbf{m T O R}$ Expression
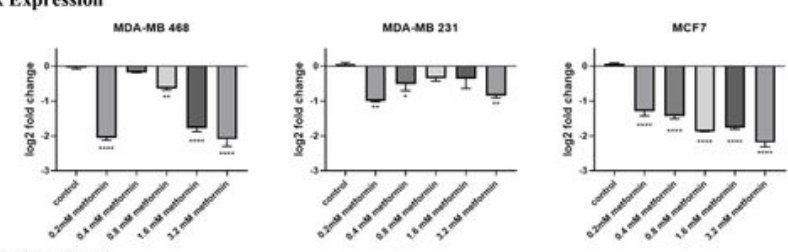

C) TOP2A Expression
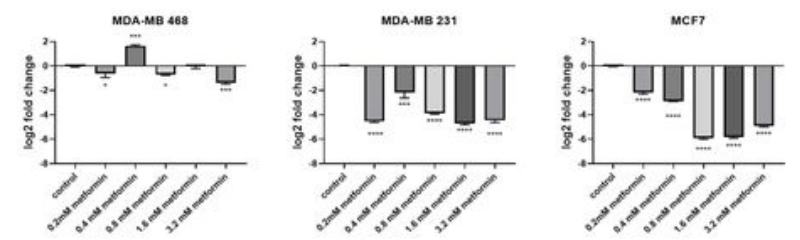

D) CCND1 Expression
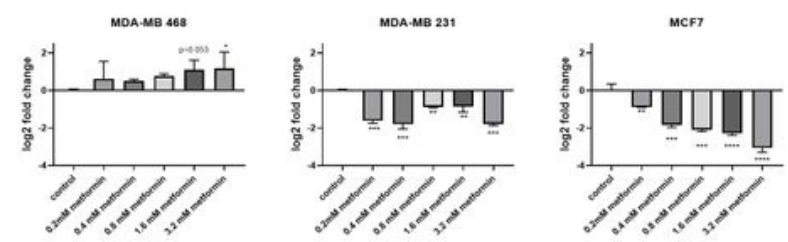

E) ZEB1 Expression
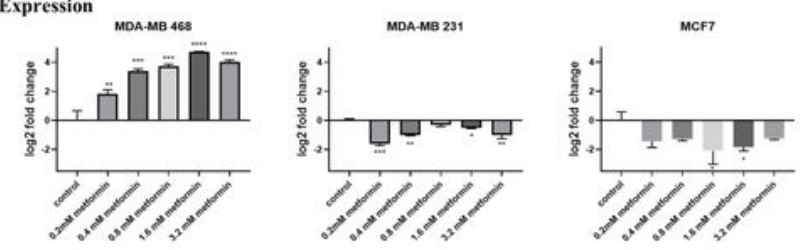

F) VIM Expression
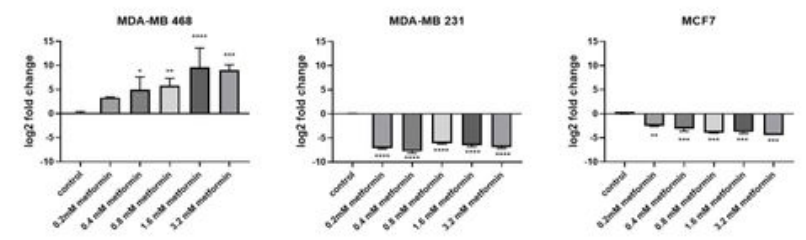

G) MMP9 Expression
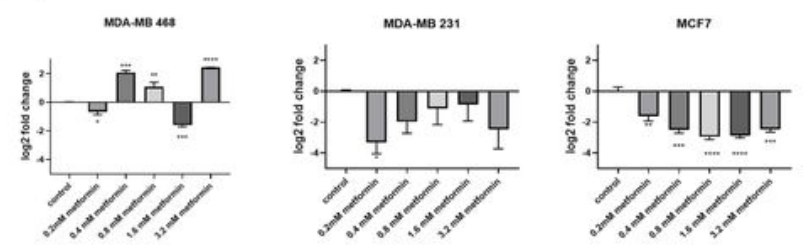

\section{Figure 1}

The qPCR analysis showed that TNBC cells treated with long term-increasing doses of metformin resulted in changes in mRNA expressions specifically related with proliferation and epithelialmesenchymal transition. 
A)
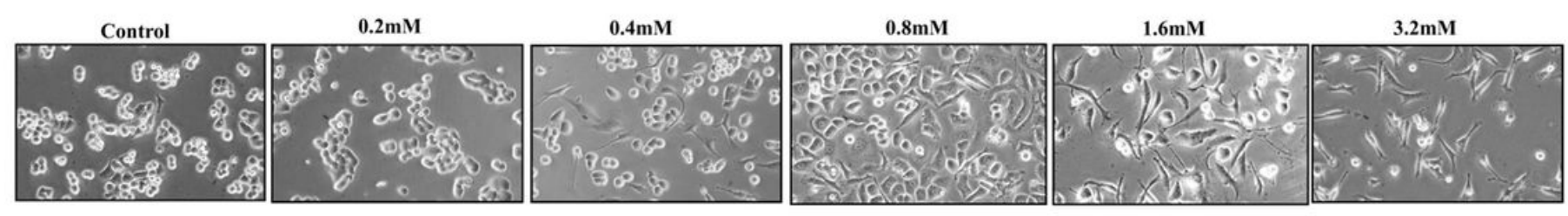

B)

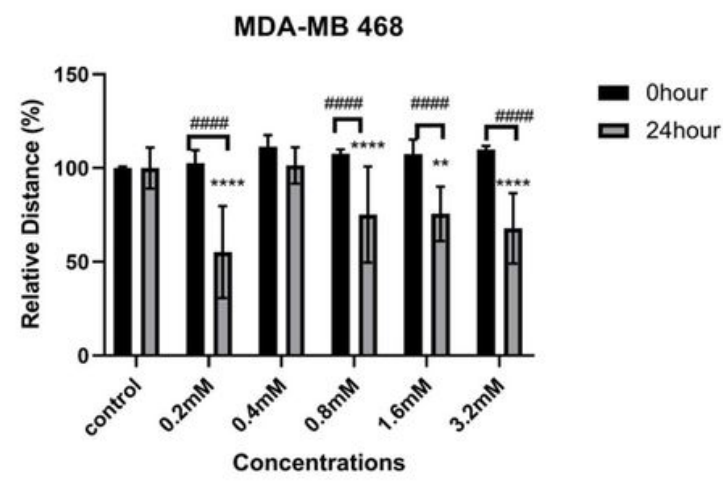

$\left.{ }^{*}\right)$ compared to 24 hour control group

(\#)comparison between time points within the same concentration groups)

Control

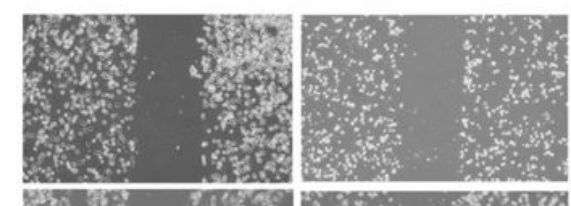

24 hour

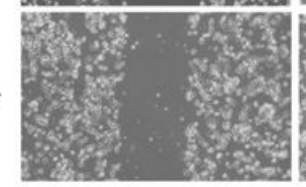

$0.2 \mathrm{mM}$

$0.4 \mathrm{mM}$
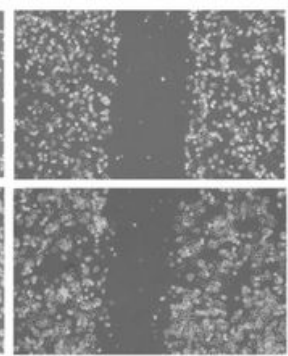

$0.8 \mathrm{mM}$

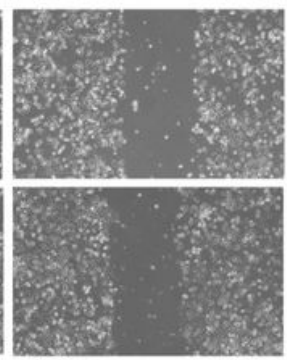

$1.6 \mathrm{mM}$

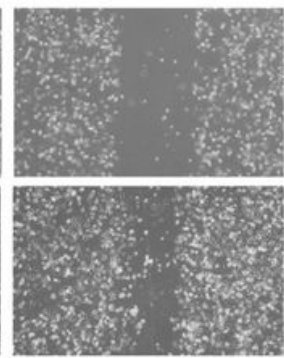

$3.2 \mathrm{mM}$

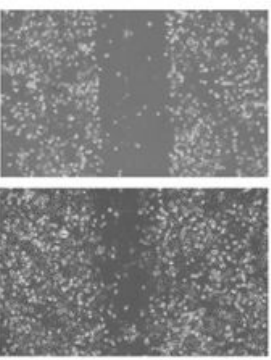

Figure 2

a: Metformin resistant cells changed their morphological structure especially in higher doses. b: Long term metformin treatment in MDA-MB 468 cells caused a significant increase of cell migration in a concentration dependent manner. 


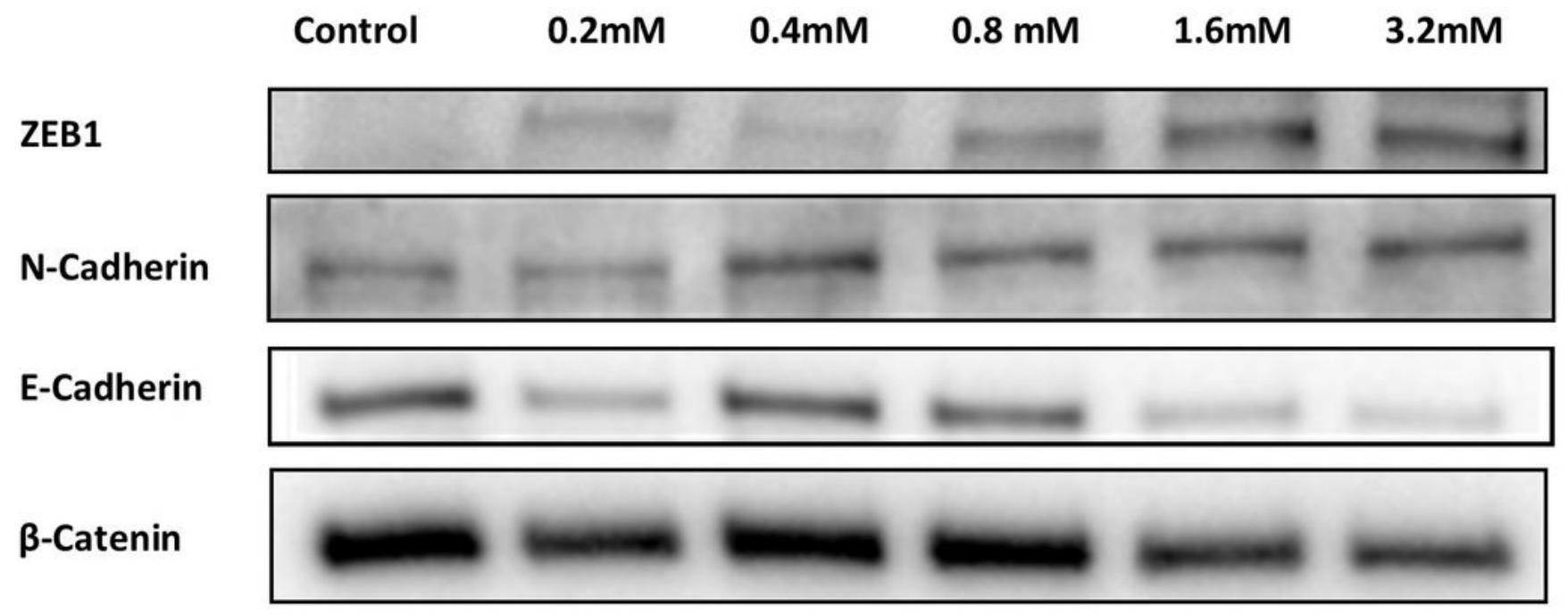

MMP2

Vimentin

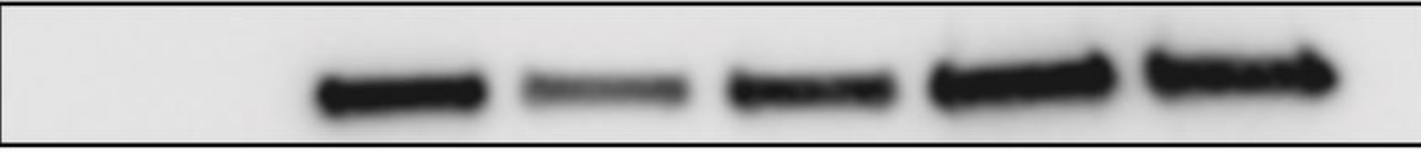

Slug

Snail

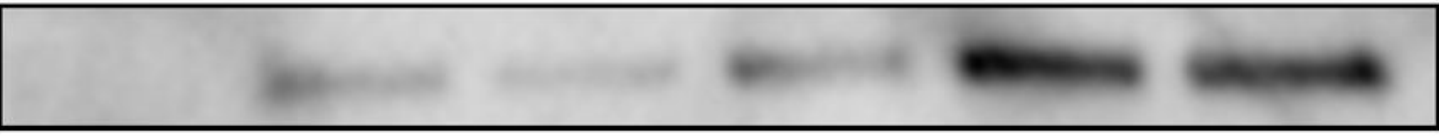

Claudin
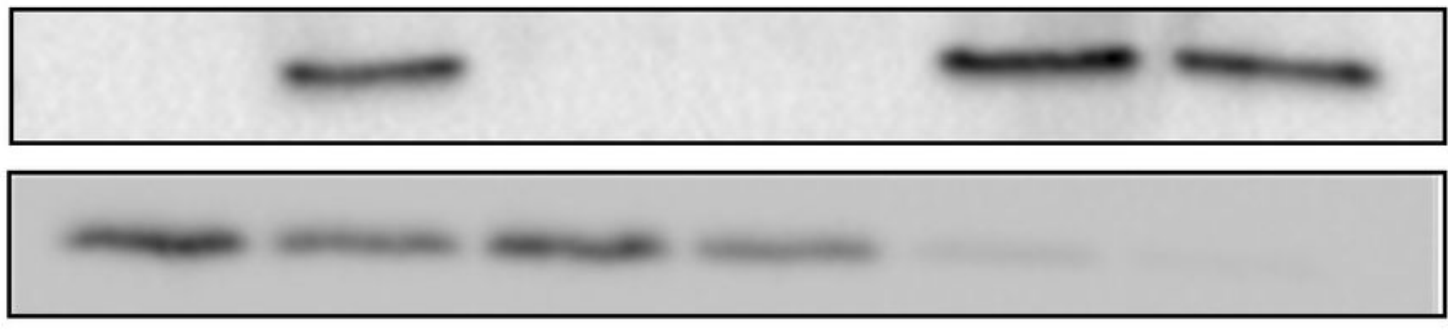

$\beta$-Actin

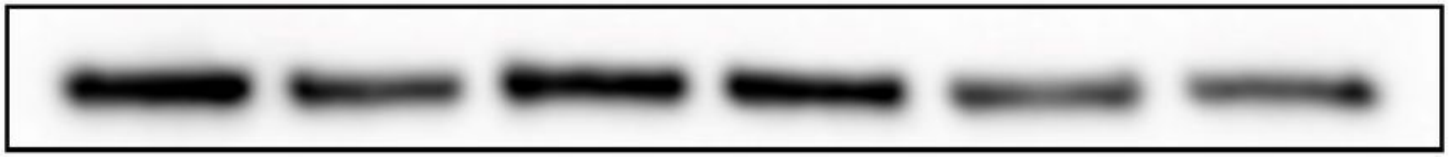

Figure 3

The altered expressions of Zeb1, N-cadherin, E-cadherin, b-catenin, MMP2, Vimentin, Slug, Snail and Claudin shows metformin resistant MDA-MB 468 cells have metastatic capacity. 

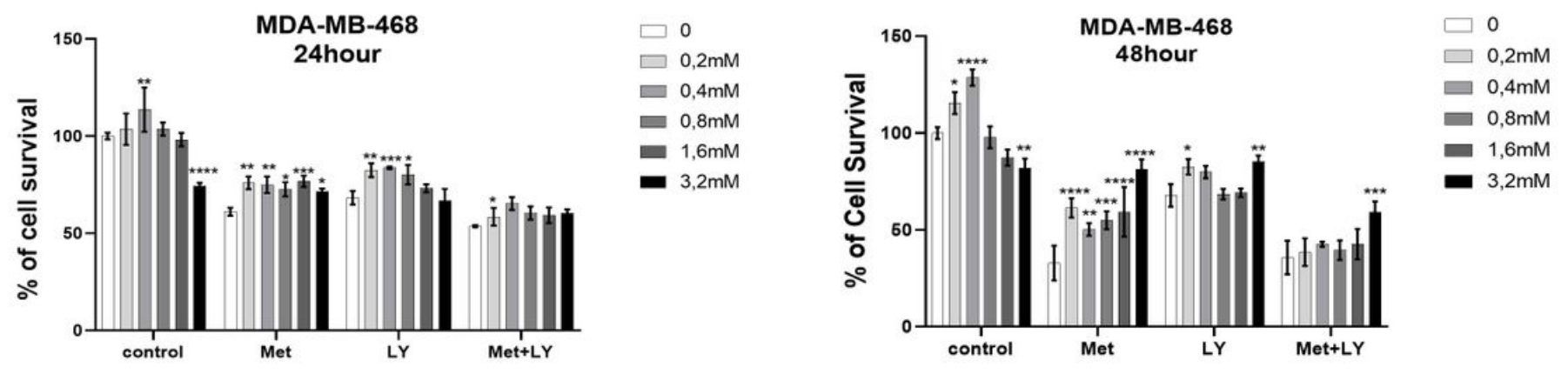

Figure 4

Effects of metformin, LY294002 and their combination on metformin resistant MDA-MB 468 cells for 24 and 48 hours. * represents comparisons within each treatment group compared to their corresponding control, $p<0,05$. Each value represents the mean $\pm S D$ of six replicates $(n=6)$.

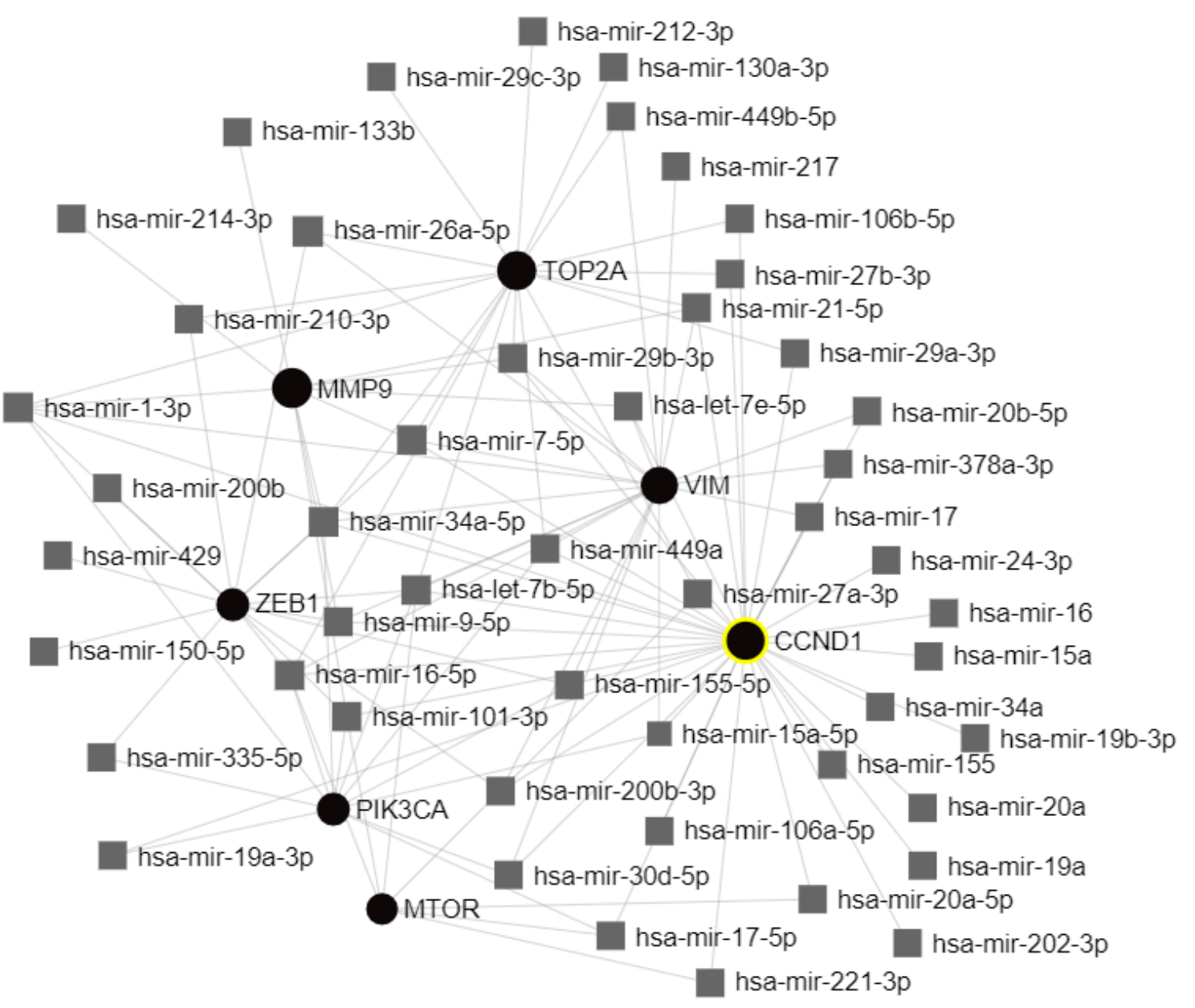


Figure 5

Shared microRNAs affected by the differentially expressed genes in met-R MDA-MB-468 cells (Squares for miRNAs; circles for altered genes)

\section{Supplementary Files}

This is a list of supplementary files associated with this preprint. Click to download.

- Table1.jpg

- AdditionalFile1.jpg 